\title{
A First Principles Investigation of Mercuric Iodide: Bulk Properties and Intrinsic Defects
}

\author{
F. Ayres ${ }^{\dagger}$, L. V. C. Assali ${ }^{\dagger}$, W. V. M. Machado ${ }^{\dagger}$, and J. F. Justo ${ }^{\ddagger}$ \\ ${ }^{\dagger}$ Instituto de Física, Universidade de São Paulo, CP 66318, 05315-970, São Paulo, SP, Brazil \\ ${ }^{\ddagger}$ Escola Politécnica, Universidade de São Paulo, CP 61548, 05424-970, São Paulo, SP, Brazil
}

Received on 31 March, 2003

\begin{abstract}
We carried a theoretical investigation on the properties of mercuric iodide in the red tetragonal crystalline phase, and its respective intrinsic defects. Our calculations were performed using the ab initio spin-polarized full-potential linearized augmented plane wave method, and taking into account full atomic relaxation. The results on the structural, electronic, and optical properties were compared to available experimental data.
\end{abstract}

\section{Introduction}

Investigations on the properties in mercuric iodide $\left(\mathrm{HgI}_{2}\right)$, as well as in other layered semiconducting materials, have increased over the last two decades $[1,2,3]$. $\mathrm{HgI}_{2}$ is potentially useful as a detector for high energy radiation (Xand $\gamma$-ray) operating at room temperature [4]. This is due to the unique combination of properties: a large band gap $(2.13 \mathrm{eV})$ and a large photoelectric absorption cross-section [5]. However, a number of limitations has precluded its widespread use as a detector. First of all, due to its bonding nature, the critical resolved shear stress is very small and the material is brittle at room temperature [6]. $\mathrm{HgI}_{2}$ also presents large concentrations of intrinsic and extrinsic defects [7]. High carrier mobility is essential for radiation detection, but defects are recombination centers that reduce considerably such mobilities. Therefore, there are two well defined fronts to investigate this material: fundamental issues, such as the electronic and optical properties, and the growing and post-growing issues, such as defect and impurity effects.

Here, we carried a theoretical investigation on the structural, electronic, and optical properties of $\mathrm{HgI}_{2}$ in the red tetragonal phase, as well as the properties of its intrinsic defects. The material was investigated considering spin-orbit effects, using an all-electron model, namely full-potential linearized augmented plane wave (FP-LAPW) method [8]. Previous theoretical investigations on the properties of mercuric iodide $[1,2,3]$ did not take into account geometry optimization, considering only the experimental lattice parameters. Our investigation is the first one to take into account such geometry optimization on all degrees of freedom.

\section{Methodology}

The calculation were carried using the WIEN97 package [9], based on the FP-LAPW method, with the generalized gradient approximation (GGA) for the exchange-correlation potential [10]. These calculations were performed by solv- ing the Kohn-Sham equations [11]. In order to describe the electronic wave-functions, the space was divided into two distinct regions: the atomic spheres, centered in the atomic positions, and the interstitial region outside the spheres. The basis set functions were expanded in plane waves outside the atomic spheres and in radial solutions of the Schrödinger's equation inside the spheres. Relativistic effects were considered for both core and valence electrons. The convergence criteria for the total energy and atomic forces were $0.1 \mathrm{mRy} /$ unit cell and $1.0 \mathrm{mRy} / \mathrm{a} . \mathrm{u}$. , respectively. For the primitive unit cell calculations, we used a plane wave cut off $\mathrm{K}_{m}=9 / \mathrm{R}$ ( $\mathrm{R}$ is the smallest atomic radii) and $6 \mathrm{k}$-points in the IBZ (Irreducible Brillouin Zone).

In the $\mathrm{HgI}_{2}$ configuration (figure 1), each $\mathrm{Hg}$ atom is tetrahedrally bonded to four I atoms, in a covalent bond. These tetrahedrals are stacked in the $\mathrm{z}$ direction, resulting in weak I-I bonds. We used the Murnaghan equation of state to get the lattice parameters ( $a$ and $c$ ) and the bulk modulus $(B)$. To get the lattice parameters, we performed a variation of the $c / a$ ratio, the volume and the internal parameters $h$ and $u$. For the density of states and optical properties, 264 $\mathrm{k}$-points in the IBZ were generated. For the optical properties, the momentum matrix elements $P_{i}=\left\langle n^{\prime} \vec{k}\left|\vec{p} \cdot \vec{e}_{i}\right| n \vec{k}\right\rangle$ between all band combinations for each k-point were obtained. The imaginary part of dielectric tensor $\varepsilon_{i j}$ is carried out by the IBZ integration [12]:

$$
\begin{array}{ll}
\operatorname{Im} & \varepsilon_{i j}(\omega)=\frac{4 \pi^{2} e^{2}}{m^{2}\left(\omega-\Delta_{c} / \hbar\right)^{2} V} \\
& \sum_{v, c, k}\left\langle v_{k}\left|p_{j}\right| c_{k}\right\rangle\left\langle c_{k}\left|p_{i}\right| v_{k}\right\rangle \delta\left(E_{c k}+\Delta_{c}-E_{v k}-\hbar \omega\right)(1)
\end{array}
$$

where $\Delta_{c}$ is the conduction band shift due to corrections in the band gap.

The 54-atom supercells were built by taking $3 a \times 3 a \times c$. For those calculations, we used $\mathrm{K}_{m}=7 / \mathrm{R}$ and IBZ integration was performed at the $\Gamma$ point. Such approximations described reasonably well the crystalline material. 


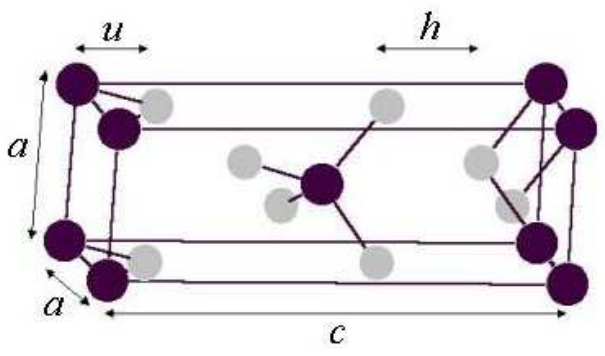

Figure 1. The primitive unit cell of red $\mathrm{HgI}_{2}$. Grey and black atoms represent $\mathrm{I}$ and $\mathrm{Hg}$ atoms, respectively. The figure shows the two lattice parameters $(a$ and $c$ ), the distance between the planes connecting the I atoms $(h)$ and the planes connecting the $\mathrm{Hg}$ and I atoms $(u)$.

\section{Results}

The electronic properties of $\mathrm{HgI}_{2}$ were obtained by using the optimized lattice and internal parameters, in contrast to previous calculations $[1,2,3]$ which considered the experimental values. The GGA for the exchange-correlation term systematically improves the results over the local density approximation in describing weak chemical bonds [13], as in the case of the I-I bonds. Our theoretical bulk modulus B = $0.22 \mathrm{Mbar}$ and the I-I bond distance $h=0.244 c$ can be compared to the experimental values of $0.44 \mathrm{Mbar}$ and $0.222 c$, respectively [14]. The lattice parameter $a$, the $c / a$ ratio, and $u$ internal parameter are respectively overestimated by $5 \%, 2.5 \%$, and $8 \%$, as shown in table I. The $E_{\text {gap }}(0.98$ $\mathrm{eV}$ ) is underestimated, as typical of density functional theory (DFT) calculations. Turner and Harmon [1] found Egap $=0.52 \mathrm{eV}$, and Solanki et al. [3] found $0.95 \mathrm{eV}$. Chang and James [2] used an empirical pseudopotential, with the band gap adjusted to the experimental value.

Table I: Theoretical and experimental values of lattice parameters $(a, c / a)$, internal parameter $u$, and band gap (Egap)

\begin{tabular}{lcccc}
\hline & $a(\AA)$ & $c / a$ & $u$ & Egap (eV) \\
\hline This work & 4.5772 & 2.9676 & $0.128 c$ & $0.98\left(1.27^{a}\right)$ \\
Expt. [15] & 4.3610 & 2.8549 & $0.139 c$ & $2.37(4.2 \mathrm{~K})[5]$ \\
\hline$a$ Without spin-orbit effects. & &
\end{tabular}

The band structure along the high-symmetry lines and the density of states are shown in figure 2 . The main contribution to the valence band comes from I $5 \mathrm{p}$ and $\mathrm{Hg} 6 \mathrm{~s}$ states. The $\mathrm{Hg} 6 s$ and I $5 p$ bonding bands are fully occupied, while the anti-bonding ones are not, indicating a covalent $\mathrm{Hg}$-I bond. The valence band width is $4.65 \mathrm{eV}$. It is also shown in the figure 2 the splitting of $5 \mathrm{~d} \mathrm{Hg}$ states by 1.10 $\mathrm{eV}$ due to the spin-orbit effects.

To investigate the optical properties of $\mathrm{HgI}_{2}[2,3]$, we computed the $\epsilon_{\|}$and the $\epsilon_{\perp}$ components of the dielectric function. The total imaginary part of dielectric function spectra of $\mathrm{HgI}_{2}\left(\left(\operatorname{Im} \epsilon_{\|}+2 \cdot \operatorname{Im} \epsilon_{\perp}\right) / 3\right)$ is shown in Fig. 3.

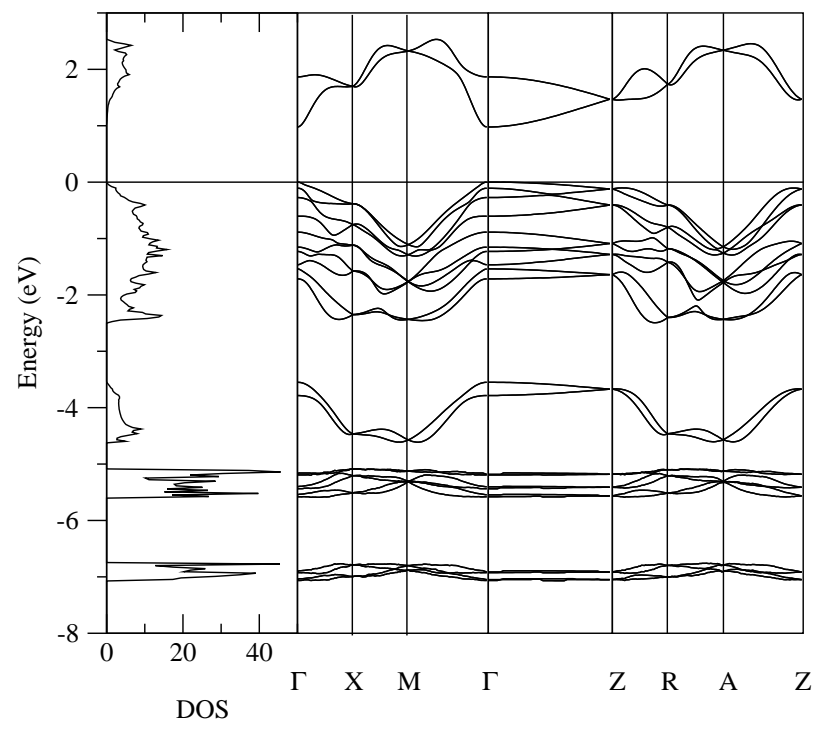

Figure 2. Band structure and density of states of $\mathrm{HgI}_{2}$.

Our optical calculations results were compared with experimental [16] and theoretical results. The first peak $(\sim 3$ $\mathrm{eV}$ ) is $\sim 1 \mathrm{eV}$ lower than the first experimental peak, but the second one $(\sim 6.5 \mathrm{eV})$ and the minimum $(5 \mathrm{eV})$ are in good agreement. The peak heights are also in good agreement with the experimental results, and gives a better description than other theoretical investigations [3].

Some $\mathrm{HgI}_{2}$ related photoluminescence (PL) lines have been identified as resulting from iodine vacancies [7]. Therefore, we carried supercell calculations on the electronic structure of the I and $\mathrm{Hg}$ vacancies. In both defects, outward relaxation was observed only on the defect firstneighboring atoms. Additionally, the $\mathrm{Hg}$ and the I vacancies presented spin $S=1 / 2$. The I vacancy related energy level is located in the upper part of the bandgap while the $\mathrm{Hg}$ vacancy related energy level is located in the lower part of the bandgap. Both defects could act as carrier traps, and consequently reducing the $\mathrm{HgI}_{2}$ detector efficiency.

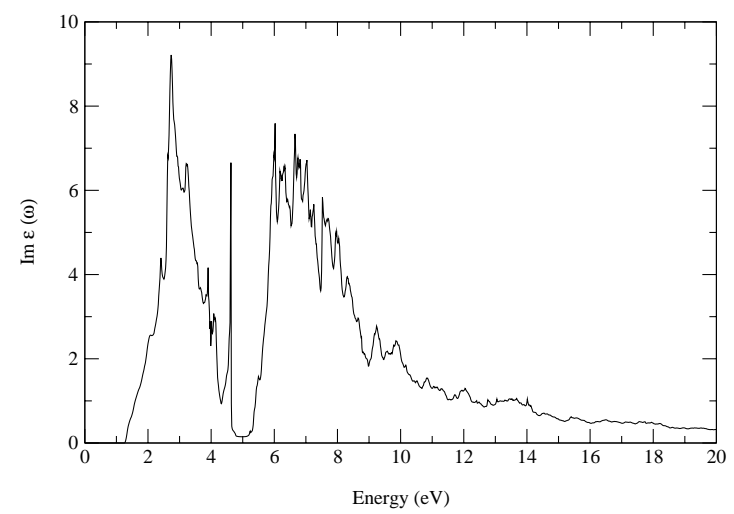

Figure 3. Imaginary part of the dielectric functions of $\mathrm{HgI}_{2}$. 


\section{Summary}

We performed the first full-potential $a b$ initio calculations of the electronic and optical properties of the red- $\mathrm{HgI}_{2}$ crystal, including the $\mathrm{Hg}$ and I vacancies. The GGA approach for the exchange-correlation potential has been shown to improve the description of the weak I-I interactions. The structural properties obtained in this work are in reasonably good agreement with experimental results. The errors might stem from the small packing fraction (large $c / a$ ) due to the weak I-I bond, which is not well described by DFT. Solanki et al. [3] have shown that a better representation of wave function leads to an improvement in the dielectric functions, which has been shown here in the comparisons of our results with theoretical [3] and experimental [16] ones. The $\mathrm{Hg}$ and I vacancies electronic structure calculations showed energy levels in the band gap region, what could be a reason of carriers trapping and, consequently, the small electron-hole mobility.

\section{Acknowledgments}

This work was supported by Brazilian agencies FAPESP and CNPq. The calculations were performed at the LCCACCE of the Universidade de São Paulo.

\section{References}

[1] D. E. Turner and B. N. Harmon, Phys. Rev. B 40, 10516 (1989).

[2] Y. C. Chang and R. B. James, Phys. Rev. B 46, 15040 (1992).
[3] A. K. Solanki, A. Kashyap, T. Nautiyal, S. Auluck, and M. A. Khan, Phys. Rev. B 55, 9215 (1997)

[4] M. Piechotka, Mater. Sci. Eng. R 18, 1 (1997).

[5] G. F. Knoll, Radiation Detection and Measurement, (John Wiley \& Sons Inc., New York), 2000.

[6] J. Marschall and F. Milstein, Appl. Phys. Lett. 62, 2784 (1993).

[7] X. J. Bao, T. E. Schlesinger, R. B. James, R. H. Stulen, C. Ortale, and A. Y. Cheng, J. Appl. Phys. 68, 86 (1990).

[8] D. J. Singh, Planewaves, Pseudopotentials and the LAPW method, (Kluver Academic, Norwell) 1994.

[9] P. Blaha, K. Schwarz, and J. Luitz, WIEN97, A Full Potential Linearized Augmented Plane Wave Package for Calculating Crystal Properties, ed: K. Schwarz, Techn. Universitat WIEN, Austria, 1999.

[10] J. P. Perdew, S. Burke, and M. Ernzerhof, Phys. Rev. Lett. 77, 3865 (1996).

[11] W. Kohn and L. J. Sham, Phys. Rev. 140, A1133 (1965).

[12] P. Puschnig and C. Ambrosch-Draxl, Phys. Rev. B 60, 7891 (1999).

[13] D. C. Patton and M. R. Pederson, Phys. Rev. A 56, R2495 (1997).

[14] N. Kuroda, T. Iwabuchi, and Y. Nishina, J. Phys. Soc. Jpn. 52, 2419 (1983).

[15] Y. F. Nicolau, M. Dupuy, and Z. Kabsch, Nucl. Instr. Meth. Phys. Res. A 283, 149 (1989).

[16] A. Anedda, E. Grilli, M. Guzzi, F. Raga, and A. Serpi, Solid State Commun. 39, 1121 (1981). 